\title{
Influence des niveaux de fertilisation sur l'enherbement et l'efficacité des herbicides dans les parcelles d'oignon au centre du Burkina Faso
}

\author{
Ali GARANE $^{1 *}$, Djibril YONLI ${ }^{1}$, Somé KOUSSAO ${ }^{1}$, Jeanne NIKIEMA ${ }^{1}$, \\ Mamoudou TRAORE ${ }^{2}$ et Mahamadou SAWADOGO ${ }^{3}$
}

\footnotetext{
${ }^{I}$ Institut de l'Environnement et de Recherches Agricoles (INERA), Centre de recherche Environnementale, Agricole et de formation de Kamboinsé (CREAF-Kamboinsé), Département Production Végétale, 01 BP 470 Ouagadougou 01, Burkina Faso.

${ }^{2}$ Institut de l'Environnement et de Recherches Agricoles (INERA/CREAF-Kamboinsè), Centre de recherche Environnementale, Agricole et de formation de Kamboinsé.

Département Gestion des Ressources Naturelles/Système de Production, 01 BP 470 Ouagadougou 01, Burkina Faso.

${ }^{3}$ Université de Ouaga I Pr Joseph Ki - Zerbo, Unité de Formation et de Recherche en Science de la Vie et de la Terre (UO/UFR-SVT), Laboratoire de Génétique et Biotechnologie Végétale, 03 BP 7021 Ouagadougou 03, Burkina Faso.

*Auteur correspondant; E-mail: ali_garane@yahoo.fr
}

\section{RESUME}

La fertilisation de l'oignon a un impact direct sur son enherbement du faite de sa forte sensibilité à la compétition des mauvaises herbes. L'objectif de ce travail est d'étudier l'influence des niveaux de fertilisation sur l'enherbement et l'efficacité des herbicides dans les parcelles d'oignon. Le dispositif expérimental utilisé est un bloc de Fisher à 4 répétitions comprenant chacune un témoin et les formulations T2, T3, T4, T5, T6 T7, $\mathrm{T} 8$, T9 et T10. Les résultats ont montré que les flores adventices inventoriées 40 et 68 jours après le repiquage (jar) ont compté respectivement 75 et 73 espèces dont $76 \%$ et $75 \%$ sont des dicotylédones. Les taux de recouvrement au sol a été compris entre $4 \%$ et $77 \% 40$ jar, entre $8 \%$ et $96 \% 68$ jar, tandis que leur biomasse sèche a évolué entre $22,41 \mathrm{~g}$ et 78,28 g 40 jar et entre 9,6 g et 148,85 g 68 jar. Le nombre de bulbes d'oignon $\left(97,33\right.$ bulbes $\left./ 6 \mathrm{~m}^{2}\right)$ a été significativement plus important dans les planches fertilisées avec $300 \mathrm{~kg} \mathrm{NPK}+\mathrm{SM}$ 40 et 68 jar (T4). Cependant, les rendements ont été significativement plus élevés avec T4 (300 kg NPK+SM 40 et 68 jar), T6 (300 kg NPK+2 L Alligator) et T7 (20 t FO+1 L Select) respectifs de 3,2 kg, 3,08 kg et 3,18 $\mathrm{kg} / 6 \mathrm{~m}^{2}$. La classe de bulbe de calibre supérieur à $30 \mathrm{~mm}$ (> $30 \mathrm{~mm}$ ) a constitué $29,32 \%$ du nombre total de bulbes récoltés. Parmi celle-ci, seul 6,69\% de bulbes ont enregistré un calibre supérieur à $45 \mathrm{~mm}$.

(C) 2019 International Formulae Group. All rights reserved.

Mots clés: Oignon, herbicides, fertilisation, enherbement, productivité. 


\title{
The influence of fertilizer control on grass cover and the effectiveness of herbicides in onion plots in the Central of Burkina Faso.
}

\begin{abstract}
ABSTRAT
Onion fertilization has a direct influence on the grass cover because of the higher sensibility of weed competition. The aim of this work is to study the effect of fertilizer control on grass cover and herbicide efficacy in onion plots. The experimental design used is a randomized complete block design with 4 replications, each containing a control and the following formulations T2, T3, T4, T5, T6 T7, T8, T9 and T10. The results showed that the adventitious plants inventoried 40 and 68 days after transplanting (dat) counted respectively 75 and 73 species of which $76 \%$ and $75 \%$ are dicotyledonous. Their rate of recovery on the ground was between $4 \%$ and $77 \% 40$ days, between $8 \%$ and $96 \% 68$ dat. Dry weed biomass ranged from $22.41 \mathrm{~g}$ to $78.28 \mathrm{~g} 40$ dat and from $9.6 \mathrm{~g}$ to $148.85 \mathrm{~g} 68$ dat. The number of bulbs $\left(97.33 \mathrm{bulbs} / 6 \mathrm{~m}^{2}\right)$ was significantly higher in the plots fertilized with $300 \mathrm{~kg} \mathrm{NPK}+$ SM 40 and 68 dat (T4) and lower in the control plots (41 bulbs / $6 \mathrm{~m}^{2}$ ). However, it is the T4 treatments (300 kg NPK + SM 40 and 68 dat), T6 (300 kg NPK + 2 L Alligator) and T7 (20 t FO + 1L Select) which observed respectively significant higher yields of $3.2 \mathrm{~kg}, 3.08 \mathrm{~kg}$ and 3.18 $\mathrm{kg} / 6 \mathrm{~m}^{2}$. The bulb class that is greater than $(>30 \mathrm{~mm}$ ) constituted $29.32 \%$ of the total number of bulbs harvested. From these, only $6.69 \%$ of bulbs recorded a size greater than $45 \mathrm{~mm}$.
\end{abstract}

(C) 2019 International Formulae Group. All rights reserved.

Keywords: Onion, herbicides, fertilization, grass cover, productivity.

\section{INTRODUCTION}

L'oignon constitue la principale culture maraîchère du Burkina Faso après la tomate. La production d'oignon est passée de 70000 tonnes en 2007 à 329319 tonnes (soit $43 \%$ de la production de légumes) en 2009-2010 (DPSAA, 2011; PAFASP, 2011). D'autre part, on enregistre une amélioration des revenus des producteurs (EASYPol, 2007). La fertilisation de l'oignon a un impact direct sur son l'enherbement, son rendement et son l'aptitude à la conservation (Carlson et Kirby, 2005; César et Franck, 2206; Smith et Fennimore, 2008; Uygur et al., 2010; Mario, 2015; DGESS, 2015; Zakari et al., 2015). Malgré son rôle capital dans la production de l'oignon et sa qualité, la fertilisation de l'oignon demeure toujours problématique dans la pratique. Ainsi, bien qu'au Burkina Faso les doses recommandées soient de 20-30 $\mathrm{t} / \mathrm{ha}$ pour la fumure organique et de $350 \mathrm{~kg} / \mathrm{ha}$ NPK (12-10-20 ou 14-23-14) associé à $130 \mathrm{~kg}$ d'urée, les doses $500-700 \mathrm{~kg} / \mathrm{ha}$ contre 400 à $500 \mathrm{~kg}$ d'urée dosé à $46 \%$ d'azote sont couramment utilisés (Gouyahali, 2016). Ces doses fortes d'engrais minéraux et la matière organique très souvent non décomposée donc pourvue d'un fort pouvoir séminal d'une part, favorise les mauvaises herbes qui trouvent les conditions idéales pour leur développement (Blackshaw et al., 2004; Stahnke, 2008; Mario, 2015). D'autre part, ces doses excessives combinées à l'irrigation tardive accroissent le calibre du bulbe et l'abondance du suc du col de l'oignon rendant difficile le séchage et la conservation des bulbes (Assane Dagna, 2006; Nasreen et al., 2007; Kurtz, 2013; Moussa, 2013). L'inadaptation des techniques de fertilisation, des doses appliquées et les difficultés de lutte contre les mauvaises herbes dans les parcelles d'oignon sont des défis à relever afin d'améliorer le rendement et la qualité du bulbe (Ghosheh, 2004; Qasem, 2006). Comment optimiser la fertilisation de l'oignon pour une production durable, de meilleure qualité sans accroitre l'enherbement de la parcelle. La présente étude a pour objectif d'évaluer l'influence des niveaux de fertilisation sur l'enherbement de l'oignon et l'efficacité des herbicides employés. D'identifier les composantes appropriées pour une approche de lutte intégrée contre les adventices de l'oignon au Centre du Burkina Faso. 


\section{MATERIEL ET METHODES}

\section{Description du milieu biogéographique}

Le village de Kamboinsé est localité à une douzaine de kilomètres au Nord de Ouagadougou sur l'axe Ouaga-Kongoussi. Ce village reçoit une pluviométrie annuelle de $700 \mathrm{~mm}$, Ainsi, la zone agroécologique à laquelle il appartient est le secteur nordsoudanien situé entre les latitudes $13^{\circ} \mathrm{N}$ et $11^{\circ} 30 \mathrm{~N}$ avec des précipitations annuelles maximales de 750 à $1000 \mathrm{~mm}$ (Thiombiano, 2005; Ngailo et al., 2013). Les sols sont de types ferrugineux remaniés et sont, de par leur situation topographique très sensible à l'érosion hydrique. C'est la zone par excellence de tous les types de savanes avec une légère dominance des savanes arbustives et arborées. Le tapis herbacé est surtout formé de plusieurs espèces de dicotylédones et de graminées, Parmi les graminées vivaces citons Imperata cylindrica, auxquelles s'ajoutent plusieurs espèces de cypéracée, la strate arborée est composée d'essences dépassant rarement $20 \mathrm{~m}$ de haut,

\section{Matériel expérimental}

Matériel végétal utilisé est constitué par la variété d'oignon Violet de Galmi de cycle court (120 jours), Le rendement potentiel varie entre 30 et 80 t/ha.

Les engrais NPK (15-15-15) l'urée (46\%) ainsi que du fumier d'étable bien décomposé ont été utilisés. Un herbicide du nom commercial Alligator $400 \mathrm{EC}$ dont la matière active est le Pendimethaline $400 \mathrm{~g} / \mathrm{l}$ a été utilisé en prélevée à la dose recommandée de $2 \mathrm{~L} / \mathrm{ha}$ et un autre herbicide du nom commercial Select 120 EC ayant le Clethodim $120 \mathrm{~g} / \mathrm{l}$ comme matière active a été appliqué en post-levée à la dose recommandée de 1 L/ha.

\section{Dispositif expérimental}

Le dispositif expérimental utilisé est un bloc Fisher (complètement randomisé) constitué de quatre répétitions et de dix traitements dont un témoin (T1) et les formulations T2 (SM 40; 68 jar), T3 (20 t FO + SM 40; 68 jar), T4 (300 kg NPK+SM 40; 68 jar), T5 (20 t FO+2 L/ha Alligator), T6 (300 $\mathrm{kg} \mathrm{NPK}+2 \mathrm{~L}$ Alligator), T7 $(20 \mathrm{t} \mathrm{FO}+1 \mathrm{~L}$ Select), T8 (300 kg NPK+1 L Select), T9 (20 t FO+2 L Alligator+SM 90), T10 (300 kg NPK+2 L Alligator+SM 90). La parcelle élémentaire est une planche de 4,0 $\mathrm{m}$ de long sur $1,5 \mathrm{~m}$ de large soit $\left(6 \mathrm{~m}^{2}\right)$ de surface par traitement au nombre total de $40(10 \times 4$ blocs). L'écart est de $0,5 \mathrm{~m}$ entre les planches et de $1 \mathrm{~m}$ entre les blocs au nombre de 4 . La superficie totale est de $400,5 \mathrm{~m}^{2}(44,5 \mathrm{~m} \times 9$ $\mathrm{m})$ sans les bordures, pour une superficie utile de $240 \mathrm{~m}^{2}\left(6 \mathrm{~m}^{2} \times 40\right)$. Les plants d'oignon ont été repiqués suivants les écartements de 20 $\mathrm{cm}$ entre les lignes et $15 \mathrm{~cm}$ entre les plants $(20 \mathrm{~cm} \times 15 \mathrm{~cm})$. Au repiquage, la densité est de 333333 plants à l'hectare ou de 200 plants par planche de $6 \mathrm{~m}^{2}$ de surface. La Figure 1 schématise le dispositif de l'essai, Des traitements ont été appliqués à chaque parcelle élémentaire (Tableau 1).

\section{Conduite de l'essai}

L'essai a été conduit durant la campagne maraîchère de 2014-2015 dans le village de Kamboinsé implanté sur un sol sablo-limoneux, La variété Violet de Galmi originaire du Niger (INRAN) est semée en pépinière le 26 novembre 2014 en terre désinfectée au préalable à la chaleur contre les maladies et ravageurs de l'oignon et une meilleure prise en charge phytosanitaire des plantules (Davis et al., 2010; Conn et al., 2012). La levée a été générale 15 jours après semis soit, le 11 décembre 2014, Les plantules d'oignon ont été repiquées le 9 janvier 2015, soit environ 43 jours après le semis. La photopériode a été de 11 h 28 min au semis en pépinière, de $11 \mathrm{~h} 24$ min à la levée générale et de $11 \mathrm{~h} 26 \mathrm{~min}$ au repiquage de l'oignon, La bulbification a commencée lorsque la photopériode était de $11 \mathrm{~h} 41 \mathrm{~min}$, L'oignon est très sensible à la longueur du jour (Currah, 2002). Les variétés de jours courts avec un besoin de 8 à 12 heures de lumière par jour pour la bulbification sont les mieux adaptées dans les conditions de culture d'Afrique tropicale (De Lannoy, 2001). Pendant le test, les températures moyennes ont été de $29,2{ }^{\circ} \mathrm{C}$ 
à l'installation de la pépinière, au repiquage de l'oignon de $26,1^{\circ} \mathrm{C}$, à la fin maturation de l'oignon de $30,7{ }^{\circ} \mathrm{C}$. La variation de températures a été de $6,7^{\circ} \mathrm{C}$ au cours du cycle de l'oignon, Le mois de mars a été le plus chaud du cycle avec $32,5^{\circ} \mathrm{C}$ et le mois de décembre le plus froid avec $26 \mathrm{oC}$, En comparaison des températures moyennes enregistrées dans la zone de Ouagadougou (minimale moyenne $24,7{ }^{\circ} \mathrm{C}$ en Janvier, maximale moyenne $32,4{ }^{\circ} \mathrm{C}$ en avril et une variation de $7,7^{\circ} \mathrm{C}$ sur l'année), la période de végétation de l'oignon a été par sa température relativement chaude Ces conditions climatiques idéales ont un impact positif sur la croissance, le développement et le rendement général de la variété (Messiaen et Rouamba, 2004).

\section{Entretien}

Les applications de l'herbicide de prélevée Alligator $400 \mathrm{EC}$, matière active la Pendimethaline $400 \mathrm{~g} / \mathrm{l}$ dose $2 \mathrm{~L} / \mathrm{ha}$ et de l'herbicide de post-levée Seclect 120 EC ayant la Clethodim $120 \mathrm{~g} / \mathrm{l}$ comme matière active dose $1 \mathrm{~L} / \mathrm{ha}$ ont été effectuées le 14 janvier et le 28n fevrier 2015 respectivement. Le premier et le deuxième sarclage ont été réalisés respectivement le 19 février et le 19 mars 2015 , soit le $40^{\text {ème }}$ et $68^{\text {ème }}$ jar dans les traitements 2 et 3 .

La fertilisation adoptée a consisté à un apport de fumure de fonds composée du fumier d'étable bien décomposé mais également de la fumure minérale le NPK (1515-15) apportées au labour suivant le traitement. La fumure de fonds composée de $20 \mathrm{t} / \mathrm{ha}$ de fumier, soit $12 \mathrm{~kg}$ de fumiers bien décomposé par planche de $6 \mathrm{~m}^{2}$; également, $350 \mathrm{~kg} / \mathrm{ha}$ de NPK, soit $208 \mathrm{~g}$ par planche de 6 $\mathrm{m}^{2}$ est apportée au cours de la préparation du sol. La fumure d'entretien de $70 \mathrm{~kg} / \mathrm{ha}$ d'urée, soit $42 \mathrm{~g} /$ planche de $6 \mathrm{~m}^{2}$ est appliquée trois semaines après repiquage et une deuxième fraction de $60 \mathrm{~kg} / \mathrm{ha}$, soit $36 \mathrm{~g} / \mathrm{planche}$ de $6 \mathrm{~m}^{2}$ un mois après la première application.

Le Décis (Deltaméthrine 12,5g/l) a été utilisé comme insecticide en cours de végétation, L'irrigation a été implantée sur un sol argilo-sableux pour la période sèche, 2 fois/semaines.

\section{Paramètres mesurés \\ Analyse de la flore}

L'inventaire des espèces a été réalisé pendant la végétation de l'oignon, La technique du «tour de champs» qui consiste à parcourir la parcelle dans toutes les directions et à recenser toutes les espèces rencontrées de plantes a été choisie (Aké Assi, 2002). Dans chaque planche, pour chacune des traitements le nombre d'espèces et leur taux de recouvrement de la parcelle ont été estimé. Les espèces inconnues sur le terrain ont été identifiées à partir des Flores de Merlier et Montegut (1982) et de Le Bourgeois et Merlier (1995).

\section{Observations et mesures}

La biomasse sèche et la densité des adventices ont été déterminées sur des placettes de $0,25 \mathrm{~m}^{2}(0,5 \mathrm{~m} \times 0,5 \mathrm{~m})$, Après arrachage manuel des adventices, la biomasse a été évaluée par la pesée de la matière sèche des parties ariennes après séchage à l'étuve pendant $24 \mathrm{~h}$ à une température de $95^{\circ} \mathrm{C}$. A la récolte: les nombres et les poids de bulbes récoltés ont été estimés, par comptage et par pesé de bulbes; le calibrage moyen des bulbes a été déterminé, pour cela, les calibres ont été catégorisés par la mesure du diamètre transversal à l'aide du fer à coulisse (modèle Vernier et Callip); également les rendements bulbes ont été calculés par traitement.

\section{Analyses statistiques}

Le traitement des données a été réalisé à l'aide du logiciel Excel 2013. Une analyse de variance (ANOVA) grâce au test de Newman Keuls a été réalisée pour l'ensemble des variables. La comparaison des moyennes a été faite selon la méthode du test de STUDENT (ppds) au seuil de 5\%. 


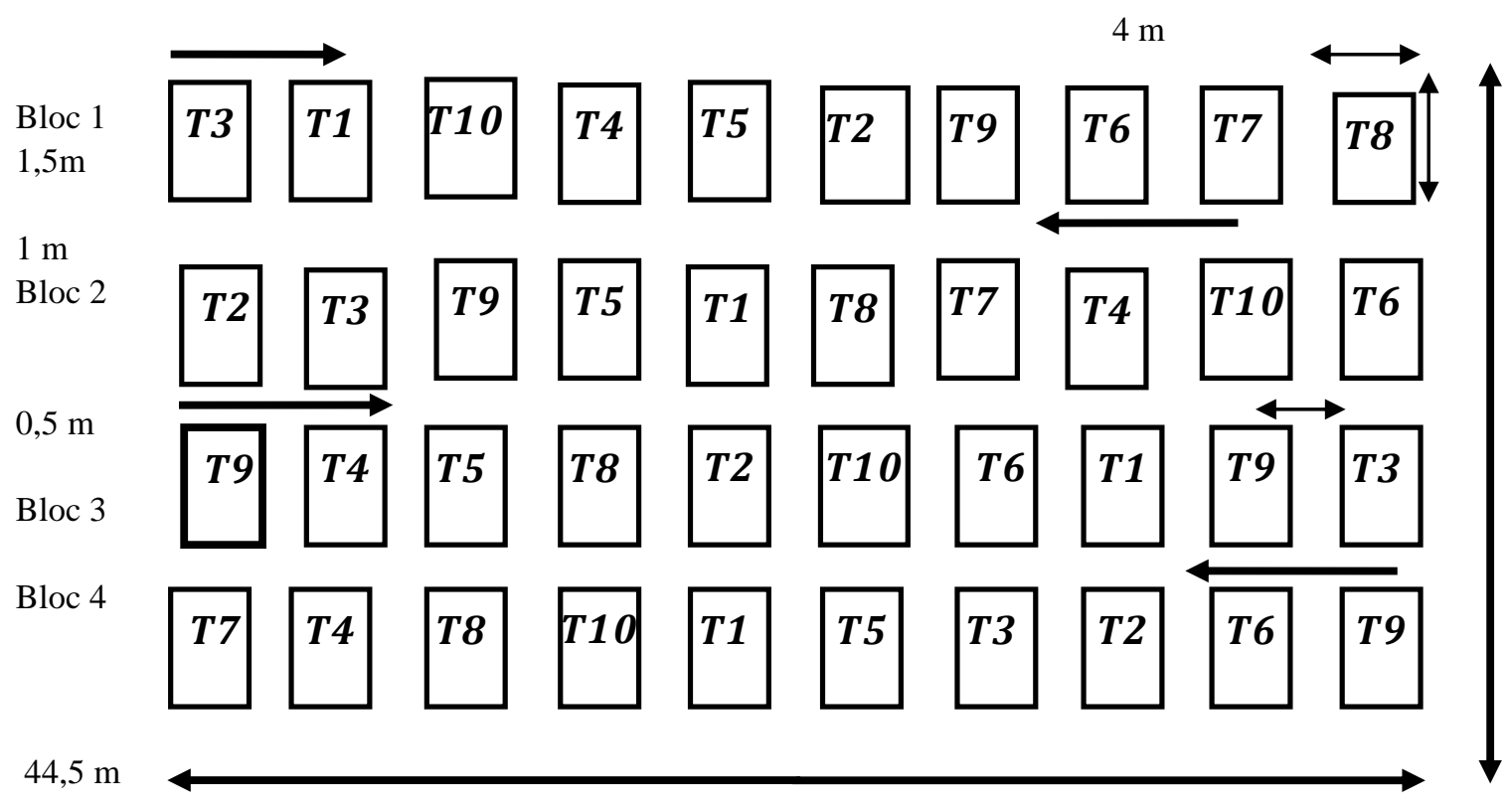

Figure 1: Dispositif expérimental utilisé.

Tableau 1: Traitements appliqués aux expérimentations et leur formulation.

\begin{tabular}{|c|c|}
\hline Traitement & Formulation \\
\hline T1 (Témoin) & Les parcelles n'ont reçu aucun entretien (sans fertilisation, ni désherbage) \\
\hline $\mathrm{T} 2$ & Sarclage manuel 40 et 68 jour après repiquage (SM 40; 68 jar) \\
\hline $\mathrm{T} 3$ & $\begin{array}{l}20 \text { tonnes/ha de Fumure organique }+ \text { Sarclage manuel au } 30 \text { et } 68 \text { jour après } \\
\text { repiquage ( } 20 \text { t FO + SM 40; } 68 \text { jar) }\end{array}$ \\
\hline $\mathrm{T} 4$ & $\begin{array}{l}300 \mathrm{~kg} / \text { ha d'engrais NPK + Sarclage manuel } 30 \text { et } 68 \text { jour après repiquage (300 } \\
\mathrm{kg} \mathrm{NPK} \mathrm{+} \mathrm{SM} \mathrm{40;} 68 \text { jar) }\end{array}$ \\
\hline T5 & $\begin{array}{l}20 \text { tonnes/ha de Fumure organique }+ \text { l'herbicide Alligator (Pendimethaline) en } \\
\text { pré-levée à la dose de } 2 \mathrm{~L} / \mathrm{ha}(20 \mathrm{t} \mathrm{FO}+2 \mathrm{~L} / \mathrm{ha} \text { Alligator })\end{array}$ \\
\hline T6 & $\begin{array}{l}300 \mathrm{~kg} / \mathrm{ha} \text { d'engrais NPK + l'herbicide Alligator (Pendimethaline) en pré-levée } \\
\text { à la dose de } 2 \mathrm{~L} / \mathrm{ha}(300 \mathrm{~kg} \mathrm{NPK}+2 \mathrm{~L} \text { Alligator) }\end{array}$ \\
\hline $\mathrm{T} 7$ & $\begin{array}{l}20 \text { tonnes de Fumure organique + L'herbicide Select (Clethodime) appliqué en } \\
\text { post-levée à la dose de } 1 \mathrm{~L} / \mathrm{ha}(20 \mathrm{t} \mathrm{FO}+1 \mathrm{~L} \text { Select })\end{array}$ \\
\hline $\mathrm{T} 8$ & $\begin{array}{l}300 \mathrm{~kg} / \text { ha d'engrais NPK + L'herbicide Select (Clethodime) appliqué en post- } \\
\text { levée à la dose de } 1 \mathrm{~L} / \mathrm{ha}(300 \mathrm{~kg} \mathrm{NPK}+1 \mathrm{~L} \text { Select) }\end{array}$ \\
\hline T9 & $\begin{array}{l}20 \text { tonnes/ha de Fumure organique }+ \text { herbicide Alligator (Pendimethaline) en } \\
\text { pré-levée à la dose de } 2 \mathrm{~L} / \mathrm{ha}+\text { Sarclage manuel } 90 \text { jour après repiquage }(20 \mathrm{t} \\
\mathrm{FO}+2 \mathrm{~L} \text { Alligator }+\mathrm{SM} 90 \mathrm{jar})\end{array}$ \\
\hline $\mathrm{T} 10$ & $\begin{array}{l}300 \mathrm{~kg} / \text { ha d'engrais NPK + herbicide Alligator (Pendimethaline) en pré-levée à } \\
\text { la dose de } 2 \mathrm{~L} / \mathrm{ha}+\text { Sarclage manuel } 90 \text { jour après repiquage }(300 \mathrm{~kg} \mathrm{NPK}+2 \mathrm{~L} \\
\text { Alligator + SM } 90 \text { jar) }\end{array}$ \\
\hline
\end{tabular}




\section{RESULTATS}

\section{Influence des traitements sur l'état d'infestation des mauvaises herbes}

Les flores adventices inventoriées 40 et 68 jours après le repiquage (jar) comptent respectivement 75 et 73 espèces dont $76 \%$ et $75 \%$ sont des dicotylédones. Les nombres d'espèces recensées par traitement ont varié entre 11 (T6) et 29 (T4) pieds 40 jar, entre 8 (T9) et 25 pieds (T7) 63 jar tandis que leur taux de recouvrement au sol a été compris entre 4\% (T6) et 77\% (T3) 40 jar, entre 8\% et 96\% 63 jar (Tableau 2). L'analyse de variance a révélé l'existence d'une différence significative entre les traitements pour les 2 paramètres (Tableau 2). L'application à l'hectare de $20 \mathrm{t}$ fumiers et $300 \mathrm{~kg}$ NPK associée à la Pendimethaline (Alligator) en pré -levée (T5, T6) ont fortement réduit 40 jar $(\mathrm{P}<0490)$ le nombre d'adventices dans les planches comparativement à celui des planches témoins (T1) qui forment un groupe homogène avec les autres traitements. Les applications à l'hectare de $20 \mathrm{t} F O$ et de 300 kg NPK associée à l'herbicide de pré-levée plus un sarclage 90 jar (T9, T10) n'a pas permis une amélioration de ce paramètre qui a été de 13,33 espèces adventices dans les deux traitements pendant la même période. A 68 jar, pour ce paramètre, la même tendance a été observée pour les traitements $\mathrm{T} 9$ et $\mathrm{T} 10$ où l'application de $300 \mathrm{~kg} \mathrm{NPK} / \mathrm{ha}$ associée à l'herbicide de pré-levée plus un sarclage 90 jar ont fortement réduit $(\mathrm{P}<0,0490)$ le nombre d'adventices dans les planches comparativement à celui de la planche témoin qui forme un groupe homogène avec les autres traitements. En revanche, le sarclage dans les traitements $\mathrm{T} 2$ et $\mathrm{T} 4$ ou l'application de l'herbicide de pré-levée (T6) ont induit 68 jar de faibles taux de recouvrement au sol par les adventices dans les planches $(\mathrm{P}<0,0010)$. La fertilisation et l'application de l'herbicide post-levée sans le sarclage supplémentaire n'ont pas significativement réduit le taux de recouvrement des adventices qui sont demeurés élevés dans les traitements T1 (72\%), T5 (61\%), T7 (96\%) et de 95\% dans T8 (Tableau 2).
Effet des traitements sur la biomasse des mauvaises herbes

En fonction des traitements, les biomasses sèches des adventices ont varié entre 22, $41 \mathrm{~g}$ (T6) et 78,28 $\mathrm{g}$ (T7) 40 jours après le repiquage (jar) et entre $9,6 \mathrm{~g}(\mathrm{~T} 2) \mathrm{g}$ et 148,85 g (T8) 68 jar (Tableau 3). L'analyse de variance (ANOVA) révèle l'existence d'une différence significative entre les traitements par rapport aux biomasses sèches des adventices prélevées 40 jar $(\mathrm{P}<0,0450)$ et 68 jar $(\mathrm{P}<0,0010)$. Les biomasses sèches les plus élevées 40 jar. ont été obtenues dans les planches fertilisées soit avec le fumier soit avec la fumure minérale sans l'application supplémentaire d'herbicide de pré-levée (Aligator). Cependant, les biomasses des adventices obtenues ont été statistiquement équivalentes dans la planche fertilisée avec $300 \mathrm{~kg} \mathrm{NPK} / \mathrm{ha}$ associé à l'application d'herbicide de pré-levée $(43,52 \mathrm{~g})$, les sarclées manuellement 40 et 68 jour après repiquage $(35,5 \mathrm{~g})$ et les planches témoins $(42,05 \mathrm{~g})$. Les biomasses sèches des adventices enregistrées 68 jar. dans les planches fertilisées avec 300 $\mathrm{kg} \mathrm{NPK/ha} \mathrm{associé} \mathrm{à} \mathrm{l'herbicide} \mathrm{de} \mathrm{post-levée}$ $(148,85 \mathrm{~g})$ ou $20 \mathrm{t}$ de fumier associé à l'herbicide de post-levée $(86,8 \mathrm{~g})$ ont été significativement supérieures à celles des autres planches. La plus faible biomasse 68 jar a été obtenue dans la planche sarclée 40 jar $(9,60 \mathrm{~g})$.

\section{Effet des traitements sur le rendement de l'oignon}

Le nombre total de bulbes récoltés a varié en moyenne de 41 à 97,33 bulbes $/ 6 \mathrm{~m}^{2}$ en fonction des traitements (Tableau 4). Ce nombre a significativement été plus important dans les planches fertilisées avec $300 \mathrm{~kg}$ NPK plus deux sarclages manuels 40 et 68 jar (97,3 bulbes $\left./ 6 \mathrm{~m}^{2}\right)$, tandis que sur les planches témoins, ce paramètre a significativement été plus faible $\left(41\right.$ bulbes $\left./ 6 \quad \mathrm{~m}^{2}\right)$. Aucune différence significative n'a été observée entre le restant des traitements qui ont enregistré pour cette variable des valeurs statiquement identiques allant de 53,33 bulbes/6 $\mathrm{m}^{2}$ (T2) à 74,33 bulbes/ $6 \mathrm{~m}^{2}$ (T9). Cependant, ce sont les poids de bulbes récoltés dans les planches 
fertilisées avec $300 \mathrm{~kg} / \mathrm{ha} \mathrm{NPK}+2 \mathrm{SM}(3,2$ $\left.\mathrm{kg} / 6 \mathrm{~m}^{2}\right)$ ou $300 \mathrm{~kg}$ NPK plus l'herbicide de pré-levée Alligator $\left(3,08 \mathrm{~kg} / 6 \mathrm{~m}^{2}\right)$ ou encore dans la planche fertilisée avec $20 \mathrm{t}$ de $\mathrm{FO} / \mathrm{ha}$ plus l'herbicide Select en post-levée $(3,18$ $\mathrm{kg} / 6 \mathrm{~m}^{2}$ ) qui ont été significativement plus élevés. La fertilisation organique $(20 \mathrm{t} / \mathrm{ha})$ suivie de 2 sarclages manuels $\left(2,97 \mathrm{~kg} / 6 \mathrm{~m}^{2}\right)$ ou de l'herbicide Alligator ne pré-levée (2,94 $\left.\mathrm{kg} / 6 \mathrm{~m}^{2}\right)$ ou encore la fumure minérale $(300$ $\mathrm{kg}$ NPK) associée uniquement a l'herbicide Select en post-levée $\left(2,63 \mathrm{~kg} / 6 \mathrm{~m}^{2}\right)$ ou suivie d'un sarclage manuel 90 jar ont observé des valeurs moins importantes qui ont supérieures au témoin $(2,53 \mathrm{~g})$

\section{Calibrage des bulbes d'oignon}

Le calibre des bulbes récoltés est représenté dan le Tableau 5. Le nombre d'oignons de tailles $0-10 \mathrm{~mm}$ a été significativement réduit au $1 / 10$ sur la planche fertilisée avec $300 \mathrm{~kg} \mathrm{NPK} /$ ha plus l'herbicide de pré-levée (T6) comparaison avec celui de la planche témoin (10,3 bulbes) (Tableau 9). Les planches fertilisées avec $300 \mathrm{~kg}$ /ha NPK plus 2 sarclages manuels 40 et 60 jar (20,33 bulbes) ont observé la plus forte valeur. Le nombre des oignons de tailles 11-15 mm obtenus avec les traitements $\mathrm{T} 3, \mathrm{~T} 7$ et $\mathrm{T} 10$ sont similaires à celui de la planche témoin. Les traitements $\mathrm{T} 4$ et $\mathrm{T} 8$ ont engendré un nombre d'oignons de tailles 16-21 $\mathrm{mm}$ identique de 15,67 bulbes. L'application 300 $\mathrm{kg}$ par hectare d'engrais NPK associés aux 2 sarclages manuels 30 et 68 jar ont engendré un nombre d'oignons de tailles $16-21 \mathrm{~mm}$ supérieur à celui du témoin qui statistiquement équivalent à celui des 18 autres traitements. L'application à l'hectare de $20 \mathrm{t}$ de fumier et de $300 \mathrm{~kg}$ d'engrais NPK associée aux 2 sarclages manuels 30 et 68 jar ont occasionné un nombre élevé d'oignons de taille 22-30 $\mathrm{mm}$ par rapport ceux de même tailles récoltés dans la planche témoin et dans 4 autres traitements respectif de 30,67 et 25,67 bulbes $/ 6 \mathrm{~m}^{2}$. Le nombre de bulbes d'oignon de taille $31-45 \mathrm{~mm}$ a été plus faible avec le témoin. Les traitements avec fertilisant seul, ou associé aux sarclages 40, 68 jar ou encore à l'herbicide de post-levée constituent un groupe homogène. Les traitements associant la fertilisation et l'herbicide de pré-levée constituent le deuxième groupe homogène avec le nombre le plus élevé de bulbes de 31$45 \mathrm{~mm}$. L'application de $300 \mathrm{~kg} \mathrm{NPK} / \mathrm{ha}$ plus l'herbicide de pré-levée a engendré le nombre le plus élevé de taille supérieure à $45 \mathrm{~mm}$ (Tableau 5).

Tableau 2: Le nombre d'espèces d'adventices et leur taux de recouvrement au sol à 40 et 68 jours après le repiquage (jar).

\begin{tabular}{lllll}
\hline \multirow{2}{*}{ Traitement } & \multicolumn{2}{c}{ 40 jours après repiquage } & \multicolumn{2}{c}{ 68 jours après repiquage } \\
& $\begin{array}{l}\text { Nombre d'espèces } \\
\text { / Planche }\end{array}$ & $\begin{array}{l}\text { Taux recouvrement } \\
\text { (\%) / Planche }\end{array}$ & $\begin{array}{l}\text { Nombre d'espèces } \\
\text { / Planche }\end{array}$ & $\begin{array}{l}\text { Taux recouvrement } \\
\text { (\%) / Planche }\end{array}$ \\
\hline T1 (Témoin) & $22,67 \mathrm{abc}$ & $65,00 \mathrm{ab}$ & $23,00 \mathrm{abc}$ & $71,67 \mathrm{abc}$ \\
T2 & $19,67 \mathrm{bcdef}$ & $40,33 \mathrm{abcde}$ & $19,00 \mathrm{abcde}$ & $10,33 \mathrm{f}$ \\
T3 & $22,67 \mathrm{abc}$ & $76,67 \mathrm{a}$ & $21,00 \mathrm{abcd}$ & $25,00 \mathrm{def}$ \\
T4 & $21,00 \mathrm{abcde}$ & $48,33 \mathrm{abc}$ & $16,00 \mathrm{abcdef}$ & $12,67 \mathrm{f}$ \\
T5 & 12,67efgh & $43,33 \mathrm{abcd}$ & $9,33 \mathrm{ef}$ & $61,00 \mathrm{abcd}$ \\
T6 & 11,00fgh & $3,67 \mathrm{ef}$ & $16,33 \mathrm{abcdef}$ & $8,00 \mathrm{f}$ \\
T7 & 29,67a & $65,00 \mathrm{ab}$ & $25,00 \mathrm{a}$ & $96,00 \mathrm{a}$ \\
T8 & $25,67 \mathrm{ab}$ & $68,33 \mathrm{a}$ & $24,33 \mathrm{abc}$ & $95,00 \mathrm{a}$ \\
T9: & $13,33 \mathrm{defgh}$ & $26,67 \mathrm{cdef}$ & $8,00 \mathrm{f}$ & $51,67 \mathrm{bcde}$ \\
T10 & $13,33 \mathrm{defgh}$ & $30,33 \mathrm{bcdef}$ & $15,00 \mathrm{bcdef}$ & $39,00 \mathrm{cdef}$ \\
\hline Moyenne & 19,39 & 43 & 18,35 & 46,5 \\
LSD & 9,033 & 36,69 & 9,958 & 36,88 \\
\hline
\end{tabular}

A l'intérieur d'une même colonne, les moyennes suivies de la même lettre ne sont pas significativement différentes selon le test de Fisher LSD au seuil de 5\%. 
Tableau 3: Biomasses sèches des mauvaises herbes obtenues en fonction des traitements.

\begin{tabular}{|c|c|c|c|c|}
\hline Traitement & $\begin{array}{l}\text { Biomasse sèche des } \\
\text { adventices } 40 \mathrm{JAR} \\
\left(\mathrm{g} / \mathbf{0}, 25 \mathrm{~m}^{2}\right)\end{array}$ & $\begin{array}{l}\text { \% par } \\
\text { rapport au } \\
\text { témoin }\end{array}$ & $\begin{array}{l}\text { Biomasse sèche des } \\
\text { adventices } 68 \mathrm{JAR} \\
\left(\mathrm{g} / 0,25 \mathrm{~m}^{2}\right)\end{array}$ & $\begin{array}{l}\text { \% par rapport } \\
\text { au témoin }\end{array}$ \\
\hline T1(Témoin) & $42,05 \mathrm{abc}$ & 100 & $58,92 \mathrm{cdef}$ & 100 \\
\hline $\mathrm{T} 2$ & $35,50 \mathrm{abc}$ & 84,42 & $9,60 \mathrm{f}$ & 16,29 \\
\hline $\mathrm{T} 3$ & $76,41 \mathrm{ab}$ & 181,71 & $28,98 \mathrm{def}$ & 49,18 \\
\hline $\mathrm{T} 4$ & $45,94 a b c$ & 109,25 & 15,69 ef & 26,62 \\
\hline T5 & $28,98 \mathrm{bc}$ & 68,91 & $44,97 \mathrm{cdef}$ & 76,32 \\
\hline T6 & $22,41 b c$ & 53,29 & 30,46 def & 51,69 \\
\hline $\mathrm{T} 7$ & $78,25 \mathrm{a}$ & 186,08 & $86,80 \mathrm{bc}$ & 147,31 \\
\hline $\mathrm{T} 8$ & $62,54 \mathrm{abc}$ & 148,72 & $148,85 \mathrm{a}$ & 252,63 \\
\hline T9 & $33,33 \mathrm{bc}$ & 79,26 & $67,71 \mathrm{cde}$ & 114,91 \\
\hline $\mathrm{T} 10$ & $43,52 \mathrm{abc}$ & 103,49 & $32,69 \mathrm{def}$ & 55,48 \\
\hline Moyenne & 39,13 & & 77.25 & \\
\hline LSD & 43,61 & & 68,42 & \\
\hline
\end{tabular}

A l'intérieur d'une même colonne, les moyennes suivies de la même lettre ne sont pas significativement différentes selon le test de Fisher LSD au seuil de 5\%.

Tableau 4: Rendements de l'oignon en fonction des traitements.

\begin{tabular}{|c|c|c|c|c|}
\hline Traitements & $\begin{array}{l}\text { Nombre bulbes } \\
\text { sur } 6 \mathrm{~m}^{2}\end{array}$ & $\begin{array}{l}\text { \% Nombre bulbes par } \\
\text { rapport au témoin }\end{array}$ & $\begin{array}{l}\text { Poids bulbes sur } \\
6 \mathrm{~m}^{2}\left(\mathrm{~kg} / 6 \mathrm{~m}^{2}\right)\end{array}$ & $\begin{array}{l}\text { \% poids bulbes par } \\
\text { rapport au témoin }\end{array}$ \\
\hline T1 (Témoin) & $41,00 \mathrm{bc}$ & 100 & 2,38 cde & 100 \\
\hline $\mathrm{T} 2$ & $53,33 \mathrm{abc}$ & 130,07 & $2,33 \mathrm{de}$ & 97,89 \\
\hline $\mathrm{T} 3$ & $81,67 \mathrm{abc}$ & 199,19 & $2,97 \mathrm{abcd}$ & 124,78 \\
\hline $\mathrm{T} 4$ & 97,33 a & 237,39 & $3,20 \mathrm{ab}$ & 134,45 \\
\hline $\mathrm{T} 5$ & $58,00 \mathrm{abc}$ & 141,46 & 2,94 abcde & 123,52 \\
\hline T6 & $57,33 \mathrm{abc}$ & 139,82 & $3,08 \mathrm{abcd}$ & 129,41 \\
\hline $\mathrm{T} 7$ & $49,00 \mathrm{abc}$ & 119,51 & $3,18 \mathrm{abc}$ & 133,61 \\
\hline $\mathrm{T} 8$ & $54,67 \mathrm{abc}$ & 133,34 & 2,63 abcde & 110,50 \\
\hline T9 & $74,33 \mathrm{abc}$ & 181,29 & 2,45 bcde & 102,94 \\
\hline $\mathrm{T} 10$ & $60,00 \mathrm{abc}$ & 146,34 & 2,51 abcde & 105,46 \\
\hline Moyenne & 62,66 & & 2,76 & \\
\hline LSD & 44,58 & & 0,7956 & \\
\hline
\end{tabular}

A l'intérieur d'une même colonne, les moyennes suivies de la même lettre ne sont pas significativement différentes selon le test de Fisher LSD au seuil de 5\%. 
Tableau 5: Le nombre de bulbes d'oignons en fonction de leur taille et des traitements.

\begin{tabular}{llllll}
\hline Traitement & $\begin{array}{l}\text { Taille bulbes } \\
(\mathbf{0 - 1 5} \mathbf{~ m m}\end{array}$ & $\begin{array}{l}\text { Taille bulbes } \\
(\mathbf{1 6 - 2 1} \mathbf{m m})\end{array}$ & $\begin{array}{l}\text { Taille bulbe } \\
\mathbf{( 2 2 - 3 0} \mathbf{~ m m})\end{array}$ & $\begin{array}{l}\text { Taille bulbes } \\
\mathbf{( 3 1 - 4 5} \mathbf{~ m m})\end{array}$ & $\begin{array}{l}\text { Taille bulbes } \\
(>\mathbf{4 5} \mathbf{~ m m})\end{array}$ \\
\hline T1(Témoin) & $19,66 \mathrm{abc}$ & $8,67 \mathrm{~cd}$ & $8,67 \mathrm{bc}$ & $0,67 \mathrm{~h}$ & $0,00 \mathrm{e}$ \\
T2 & $20,34 \mathrm{abc}$ & $7,00 \mathrm{~cd}$ & $18,67 \mathrm{abc}$ & $9,00 \mathrm{cdefgh}$ & $0,33 \mathrm{de}$ \\
T3 & $14,00 \mathrm{abc}$ & $18,00 \mathrm{bc}$ & $30,67 \mathrm{ab}$ & $15,00 \mathrm{abcdefgh}$ & $0,00 \mathrm{e}$ \\
T4 & 36,00a & $30,00 \mathrm{a}$ & $25,67 \mathrm{ab}$ & $8,00 \mathrm{defgh}$ & $0,00 \mathrm{e}$ \\
T5 & $7,00 \mathrm{bc}$ & $13,33 \mathrm{bcd}$ & $11,33 \mathrm{abc}$ & $22,67 \mathrm{abcdefg}$ & $3,67 \mathrm{cde}$ \\
T6 & $1,66 \mathrm{c}$ & $3,00 \mathrm{~d}$ & $8,67 \mathrm{bc}$ & $23,67 \mathrm{abcdef}$ & $20,33 \mathrm{a}$ \\
T7 & $13,33 \mathrm{abc}$ & $12,67 \mathrm{bcd}$ & $15,67 \mathrm{abc}$ & $6,67 \mathrm{defgh}$ & $0,33 \mathrm{de}$ \\
T8 & $29,00 \mathrm{ab}$ & $9,00 \mathrm{~cd}$ & $12,00 \mathrm{abc}$ & $4,00 \mathrm{efgh}$ & $0,67 \mathrm{cde}$ \\
T9 & $9,67 \mathrm{bc}$ & $15,00 \mathrm{bc}$ & $24,33 \mathrm{abc}$ & $27,00 \mathrm{abcd}$ & $5,00 \mathrm{bcde}$ \\
T10 & $14,00 \mathrm{abc}$ & $13,33 \mathrm{bcd}$ & $18,33 \mathrm{abc}$ & $33,33 \mathrm{ab}$ & $14,00 \mathrm{ab}$ \\
\hline Moyenne & 16,46 & 13,83 & 17,5 & 15,3 & 3,76 \\
LSD & & 11,66 & 19,80 & 21,50 & 9,16 \\
\hline
\end{tabular}

A l'intérieur d'une même colonne, les moyennes suivies de la même lettre ne sont pas significativement différentes selon le test de Fisher LSD au seuil de 5\%.

\section{DISCUSSION}

\section{Nombre d'espèces adventices et taux de recouvrement au sol}

La flore adventice inventoriée 40 et 68 jours après le repiquage (jar) a comptée respectivement 75 et 73 espèces dont $76 \%$ et $75 \%$ sont des dicotylédones. Le nombre d'espèces reste plus important que celui signalé dans la même zone par Garané et al. (2017a) qui ont inventorié 24 espèces appartenant à 22 genres et 16 familles. Les Dicotylédones ont été dominantes avec 20 espèces $(83,33 \%)$. De même, la flore adventice dénombrée a été plus importante comparativement à la flore associée à l'oignon en végétation au Nord-Est du Bénin, qui a enregistrée 17 espèces réparties dans six familles décrit par Bello et al. (2013). Toutefois le nombre d'espèce enregistré a été moins important par rapport à celui signalé par Garané et al. (2017b) dans les cultures de la patate douce du centre du Burkina Faso qui ont répertorié 102 espèces. Ces derniers ont observé un taux moins important de dicotylédones soit de $68,63 \%$. A la première date de relevé 40 jar, on dénombre entre 11 et 29 espèces différentes par traitement. Au cours de la deuxième date de relevé (68 jar), ces chiffres ont évolué pour atteindre 8 à 25 espèces par traitement. Cette variation de la biodiversité floristique est assez faible car il ya 4 espèces de moins que la flore initiale. La chute du nombre d'espèces adventices entre la première et deuxième date de relevé peut signifier l'absence d'espèces nouvelles mais surtout la présence d'espèces hâtives dont la germination a lieu plus tôt pendant le cycle de culture de l'oignon (Ahanchédé, 2000). Selon Hüsrev et Doğan (2003), la composition de la flore des parcelles d'oignon serait fortement dénaturée par les pratiques de contrôle de l'enherbement non diversifiées, les rotations, la contamination des semences et la fertilisation. Aussi, pour Hodonou et Savi (2001) et Bello (2012) la maîtrise des mauvaises herbes de l'oignon par des 
méthodes améliorées de lutte était toujours une préoccupation des producteurs. Le sarclage manuel reste la méthode de lutte la plus courante employée au détriment du contrôle chimique pour le contrôle mauvaises herbes dans les parcelles d'oignon (Conn et al., 2012). Ces résultats corroborent avec ceux de Hodonou et Savi (2001), qui ont préconisé quatre opérations de sarclo-binages au cours du cycle cultural de l'oignon entre 15 et 30 jar, à 45 jar et à 60 jar en absence d'utilisation d'herbicides pour permettre une bonne croissance et développement de l'oignon et réduire la densité et la biomasse adventices à leurs plus faibles valeurs après 75 jar. L'évaluation du recouvrement des adventices par période de relevé montre pour les parcelles témoins des taux importants de recouvrement de 65,0 et $71,67 \%$ /planche à 40 et $68 \mathrm{jar}$ respectivement. Indépendamment de la période considérée, par traitement les taux de recouvrement au sol ont été compris entre $3,67-76,67 \%$ à la première date de relevé ( 40 jar) et compris $8,00-96,00 \%$ pour la date de 68 jar. Pour ces deux périodes, les pourcentages de recouvrements ont été sensiblement plus importants dans la fertilisation T7 (20 t FO + $1 \mathrm{~L}$ Select) et la fertilisation T8 $(300 \mathrm{~kg} \mathrm{NPK}+1 \mathrm{~L}$ Select $)$ respectifs de 65,0 et $96,0 \%$ /planche pour le premier et de 63,33 et 95,0\% /planche pour le second. Pour ces deux traitements (T7 et T8), les planches ont été caractérisées pendant les deux périodes par un nombre plus élevé d'espèce adventices, respective de 27 et de 25 espèces/Planches de $6 \mathrm{~m}^{2}$.

Selon l'échelle de la Commission des Essais Biologiques revue par Marnotte (1984), ces taux ont évolué de moins d'un individu/ $\mathrm{m}^{2}$ à un recouvrement de $77 \% 40$ jar et à 68 jar de moins d'un individu $/ \mathrm{m}^{2}$ à un fort recouvrement de $96 \%$. Dans les deux cas, la Clethodime appliquée en post-levée n'a pas influé positivement sur les taux de recouvrement des planches qui sont demeurés élevés pour ces deux traitements. Ces taux très importants de recouvrement du sol ont un impact négatif sur le rendement et sur la qualité des oignons récoltés dans ces traitements. En effet, la faible couverture du sol et la lenteur de croissance caractéristique en début de cycle rend l'oignon plus sensible à la compétition des mauvaises herbes (Smith and Fennimore, 2008; Mario, 2015). Selon Uygur et al. (2010), celles-ci peuvent occasionner des pertes de rendement pouvant atteindre plus d'une tonne/ha. Pour réduire voire annihiler la compétition précoce des adventices et lutter contre les espèces plus résistantes, difficiles a éradiquer, il est primordial de trouver des méthodes améliorées de lutte avec un recours au traitement chimique (Bello, 2012; Mario, 2015). Au contraire, le sarclage manuel 40 et 68 jar (T2), ou associé a 20 tonnes de Fumure organique (T3) ou encore a $300 \mathrm{~kg}$ par hectare d'engrais NPK (T4) ont enregistré les plus faibles taux de recouvrement respectifs de 10,$33 ; 25,0$ et $12,67 \% 68$ JAR. Seul, le traitement T6 ou la Pendimethaline est associée par hectare a $300 \mathrm{~kg}$ d'engrais NPK en pré-levée a enregistré pour ce paramètre des valeurs plus faibles 3,67\% (40 jar) et $8,0 \%$ (68 jar). Aussi bien le sarclage manuel que l'herbicide Alligator (Pendimethaline) en prélevée a eu impact positif sur les taux de recouvrement des parcelles concernées.

\section{Biomasse sèche des mauvaises herbes}

A 40 jar, la biomasse enregistrée a été plus forte $\left(78,25 \mathrm{~g} / 0,25 \mathrm{~m}^{2}\right)$ sur les planches fertilisées avec 20 tonnes de fumiers associé à l'herbicide Select en post-levée. De même, dans une moindre mesure, ce paramètre a été aussi important sur les planches fertilisées avec 20 tonnes de fumiers associé à un sarclage 40 et 68 jar $\left(76,41 \mathrm{~g} / 0,25 \mathrm{~m}^{2}\right)$ ainsi que sur les planches fertilisées avec $300 \mathrm{~kg}$ par hectare d'engrais NPK combiné à l'herbicide Select en post-levée $\left(62,54 \mathrm{~g} / 0,25 \mathrm{~m}^{2}\right)$. La plus faible biomasse sèches $\left(22,41 \mathrm{~g} / 0,25 \mathrm{~m}^{2}\right)$ a été obtenue dans les planches amendées avec $300 \mathrm{~kg} / \mathrm{ha}$ d'engrais NPK plus l'herbicide Alligator en pré-levée. De part ce résultat, ce traitement reste statiquement comparable aux traitements T5 $\left(28,98 \mathrm{~g} / 0,25 \mathrm{~m}^{2}\right)$ et T9 $\left(33,336 \mathrm{~g} / 0,25 \mathrm{~m}^{2}\right)$. Comparativement au témoin sans fertilisation, l'application de 20 t/ha de fumiers plus l'herbicide Select en post-levée (T7) a provoqué une forte croissance de la biomasse 
sèche de 86,08\%; tandis que l'apport de 20 t/ha de fumiers plus 2 sarclages (T3) augmentait la biomasse sèches de $81,71 \%$. Contrairement à ces derniers, l'utilisation de $300 \mathrm{~kg}$ par hectare d'engrais NPK associée à l'herbicide Alligator en pré-levée a contribuée à réduire de $46,71 \%$ la production de biomasse sèche dans ce traitement.

A 68 jar, ce sont d'une part les planches fertilisées avec 20 t FO + 1 L Select (T7) et d'autre part celles qui ont reçu $300 \mathrm{~kg}$ NPK associé à l'herbicide Select en post-levée (T2) qui ont observé les plus forts chiffres pour la matière sèche produite, soit 86,80 et 148,85 $\mathrm{g} / 0,25 \mathrm{~m}^{2}$ respectivement. Par rapport a la première période, les traitements $\mathrm{T} 8(300 \mathrm{~kg}$ $\mathrm{NPK}+1$ L Select $)$ et $\mathrm{T} 9(20 \mathrm{t} \mathrm{FO}+2 \mathrm{~L}$ Alligator + SM 90) ont enregistré les plus forts taux de croissance de leur biomasse sèche soit, plus du double de leur valeurs antérieurs respectives de $138 \%\left(148,85 \mathrm{~g} / 0,25 \mathrm{~m}^{2}\right)$ et $103 \%\left(67,71 \mathrm{~g} / 0,25 \mathrm{~m}^{2}\right)$ contre 62,54 et 33,33 $\mathrm{g} / 0,25 \mathrm{~m}^{2}$ respectivement 40 jar. L'utilisation de deux sarclages manuels dans le traitement T2 sans fertilisation, et les traitements $\mathrm{T} 3$ et $\mathrm{T} 4$ avec une fertilisation de 20 tonnes de fumier/ha et de $300 \mathrm{~kg}$ d'engrais NPK/ha respectivement ont très tôt influencé l'enherbement de ces parcelles se traduisant très tôt par la maitrise des biomasses sèche qui ont très peu évoluée au cours de la période suivante $\left(9,6 ; 28,98\right.$ et $\left.15,69 \mathrm{~g} / 0,25 \mathrm{~m}^{2}\right)$. Comparativement au témoin de l'essai, les traitements T9, T7 et surtout T8 ont enregistré des hausses de biomasse sèche de 14,91; 47,31 et de $105,91 \%$ respectivement à 68 jar. Cependant, une prise en compte des deux périodes de relevés montre que la plus faible production de biomasse sèche a été de 9,60 $\mathrm{g} / 0,25 \mathrm{~m}^{2}$ avec sarclage manuel au 40 et $68 \mathrm{jar}$ sans fertilisation à 68 jar et la plus forte production a été de $148,85 \mathrm{~g} / 0,25 \mathrm{~m}^{2}$ ) avec la dose $300 \mathrm{~kg}$ NPK+ 1 L Select à hectare (T8) à 68 jar.

\section{Rendement de l'oignon}

L'analyse des composantes de rendement a monté un nombre de bulbes en général très faible par traitement allant de 41 à 97,33 bulbes $/ 6 \mathrm{~m}^{2}$. Pour cette variable, les planches témoins sans fertilisation ont enregistré la plus faible valeur de 41,00 bulbes $/ 6 \mathrm{~m}^{2}$ différent statiquement des autres traitements de l'essai. Pour cette variable, les fertilisées planches avec $300 \mathrm{~kg}$ d' NPK/ha associé a un sarclage manuel 40 et 68 jour après repiquage (T4), ont observé un nombre de bulbes significativement plus important de 97,33 bulbes $/ 6 \mathrm{~m}^{2}$. Pour la grande majorité des traitements T2, T3, T5 T6, T8, T9 et dans une moindre mesure $\mathrm{T} 7$, les quantités de bulbes ont été statiquement identiques respectives de 53,$33 ; 81,67 ; 58,0 ; 57,33 ; 54,67 ; 74,33$ et 60,0 bulbes $/ 6 \mathrm{~m}^{2}$. Par rapport au témoin, les gains de rendement bulbes ont été plus importants et supérieurs et compris entre 19,51\% (T7) et $137,39 \%$ (T4).

S'agissant du poids moyen bulbes récoltés, les doses employées de fertilisants ont très peu influencé ce paramètre qui est demeuré très faible par traitement.et inférieur a $3 \mathrm{~kg} / 6 \mathrm{~m}^{2}$. Cependant, cette variable a observé une variation en fonction des quantités de bulbes récoltés dans chaque traitement. Les planches sarclées 40 et 68 jar (T2) ont enregistré le plus faible poids moyen bulbes de $2,33 \mathrm{~kg} / 6 \mathrm{~m}^{2}$, tandis que T4 $(300 \mathrm{~kg} \mathrm{NPK}+2$ $\mathrm{SM})$ avait la plus forte valeur de $3,2 \mathrm{~kg} / 6 \mathrm{~m}^{2}$. Outre ces derniers, la fertilisation avec $300 \mathrm{~kg}$ NPK/ha plus l'herbicide de pré-levée Alligator (T6) et 20 tonnes de fumier associé à l'herbicide Select en post-levé (T7) avaient des poids bulbes légèrement plus importants respectifs de 3,08 et 3,18 kg/ $6 \mathrm{~m}^{2}$. Dans ces conditions de fertilisation minérale ou organique, les rendements obtenus ont été en général très faibles pour cette variété qui en temps normal de production atteint un poids moyen bulbe de 21,7 à $150 \mathrm{~g}$ et des rendements bulbes de moyen de 45 t/ha pouvant culminer à 60 t/ha (Assane Dagna, 2006; Assogba-Komlan et al., 2007; AVRDC, 2014).

\section{Calibrage des bulbes d'oignon}

Les calibres (diamètre du bulbe à son point le plus large) de bulbes retenus ont été: 0 $-15,16-21,22-30,31-45$ et supérieur à 45 $\mathrm{mm}$. Indépendamment du traitement considéré, les bulbes récoltés ont enregistré des calibres 
faibles et parfois très faibles. La classe de bulbes de calibre $0-30 \mathrm{~mm}$ a été la plus importante, soit $70,68 \%$ du nombre total de bulbes récoltés. Cette dernière est composée en pourcentage des bulbes de calibre $0-15 \mathrm{~mm}$ $23,83 \%, 16-20 \mathrm{~mm}-26,24 \%$ et enfin de calibre $21-30 \mathrm{~mm}-19,6 \%$ qui ont été identiques statiquement. La classe de bulbes de calibre supérieur à $30 \mathrm{~mm}$ a occupé $29,32 \%$ du nombre total de bulbes récoltés. Parmi cette classe, 22,63\% des bulbes avaient un calibre compris entre 31 - $45 \mathrm{~mm}$ et très peu de bulbes ont enregistré un calibre supérieur à $45 \mathrm{~mm}$ $(6,69 \%)$. Dans la majorité des cas, la classe de bulbes de calibre supérieurs à $45 \mathrm{~mm}$ a été soit inexistante (T1, T3 et $\mathrm{T} 4$ ), soit pratiquement nulle ( $\mathrm{T} 2, \mathrm{~T} 7$ et $\mathrm{T} 4)$ ou très peu fréquente (T5 et T9) pour la plupart des traitements. Ce résultat ne peut être considéré comme satisfaisant dans la mesure où selon N'Guessan (1997), la sélection du matériel végétal et les techniques culturales sont menées afin d'obtenir des bulbes de calibre $40-60 \mathrm{~mm}$ pour le Violet de Galmi. Au cours de l'essai, indépendamment de traitements appliqués, la variété a produit seulement $6,69 \%$ de bulbes de calibre supérieur à $45 \mathrm{~mm}$. La prédominance de bulbes de petits calibres confirme la faible performance générale des différents traitements et explique le faible rendement de la variété testée. Dans les conditions de fertilisation minérale (NPK) ou organique (fumier) de l'essai, le Violet de Galmi n'a pas exprimé son caractère d'origine. Au contraire, la variété a formée $70,68 \%$ de bulbes de calibre 0 - $30 \mathrm{~mm}$ avec un taux de bulbilles de $24,83 \%$ (petit bulbe dont le calibre n'excède pas $15 \mathrm{~mm}$ ), tandis que les bulbes de calibre supérieur à $45 \mathrm{~mm}$ ont constitué seulement $6,69 \%$ de la production totale.

En effet, les doses recommandées sont de 20-30 t/ha pour la fumure organique et de $350 \mathrm{~kg} / \mathrm{ha}$ NPK (12-10-20 ou 14-23-14) associé à $130 \mathrm{~kg}$ d'urée dosé à $46 \%$ pour la fertilisation minérale au Burkina Faso dans la fertilisation de l'oignon (Conn et al., 2012).

\section{Conclusion}

Les résultats de l'étude ont montré que les flores adventices inventoriées 40 et 68 jours après le repiquage (jar) ont compté respectivement 75 et 73 espèces dont $76 \%$ et $75 \%$ sont des dicotylédones. Les nombres d'espèces recensées par traitement ont varié entre 11 et 29 pieds 40 jar, entre 8 et 25 pieds 63 jar, tandis que leur taux de recouvrement au sol a été compris entre $4 \%$ et $77 \% 40$ jar, entre $8 \%$ et $96 \% 63$ jar. Les traitements $\mathrm{T} 4$ (300 kg /ha NPK+ SM 40, 68 jar), T6 (300 kg $\mathrm{NPK}+2 \mathrm{~L}$ Alligator) et $\mathrm{T} 7(20 \mathrm{t} \mathrm{FO}+1 \mathrm{~L})$ ont présenté des rendements bulbes significativement plus importants respectifs de $3,2 \mathrm{~kg} ; 3,08 \mathrm{~kg}$ et $3,18 \mathrm{~kg} / 6 \mathrm{~m}^{2}$. Le calibrage des bulbes a montré une prédominance de la classe de bulbes de 0 - $30 \mathrm{~mm}$ qui a constitué $70,68 \%$ du nombre total de bulbes récoltés dont $24,83 \%$ de bulbilles. La classe de bulbes de calibre supérieur à $30 \mathrm{~mm}$ a occupé $29,32 \%$. Parmi celle-ci, seul 6,69\% de bulbes ont enregistré un calibre supérieur à $45 \mathrm{~mm}$. La prédominance de bulbes de petits calibres (70,68\%) confirme la faible performance générale des différents traitements et explique le faible rendement de la variété testée.

\section{CONFLIT D'INTERÊTS}

Tous les auteurs déclarent ne pas avoir un conflit d'intérêts.

\section{CONTRIBUTIONS DES AUTEURS}

AG a collecté les données, écrit le manuscrit (40\%) DJ a contribué à la conception de la méthodologie (tous les auteurs ont convenu de la méthode d'échantillonnage, de la période et du choix du site). Il contribué à la rédaction de la méthodologie lu et amélioré la section de discussion (20\%). JN et MT ont participé à écriture et à l'amélioration du manuscrit. Ils ont également contribué à la vérification linguistique, lu et amélioré la section de discussion (15\% chacun) MS a contribué à la rédaction de la méthodologie lu et amélioré la section de discussion. Il a également fourni des commentaires (10\%). 


\section{REFERENCES}

Ahanchédé A. 2000. Compétition entre mauvaises herbes et cultures cotonnière: influence du nombre de sarclages sur la biomasse et le rendement. Tropicultura, 18(3):148-151.

Aké Assi L. 2002. Flore de la Côte d'Ivoire 2: Catalogue systématique, biogéographie et écologie. Conservatoire et Jardin Botanique Genève, Suisse, p. 401.

Alessandro S, Alseny S. 2008. Note technique : Évaluation sous-régionale de la chaine de valeurs oignon échalote en Afrique de l'ouest. ATP, p. 58.

Assane Dagna M. 2006. Les effets de la réappropriation de la culture du Violet de Galmi par les producteurs d'oignon de la région de Tahoua - NIGER, sur la dynamique $\mathrm{du}$ territoire local, l'organisation sociale et économique. Thèse de doctorat Option développement rural, Université de Toulouse-Le, Toulouse, p. 281.

Assogba-Komlan FK, Bello S, Baco M. 2007. Sélection participative au champ de quelques cultivars d'oignon de jours courts pour le département de l'Alibori. Bulletin de la Recherche Agronomique du Bénin, 58: 745 - 755.

AVRDC (The World Vegetable Center) 2014. Year in Review: Develop short-day red onions and yellow onions for improved yield, extended shelf-life, and/or Stemphylium resistance. AVRDC, p.78

Bello S, Afouda OY. 2002. Rapport voyage d'échanges d'expériences entre producteurs Nigériens et Béninois au Niger. INRAB-CRAN/PADSE, Bénin, p. 37

Bello S, Ahanchédé A, Gbèhounou G, Amadji G, Aho N. 2013. Diversité floristique, ethnobotanique et taxonomie locale des mauvaises herbes de l'oignon au Nordest du Bénin. Tropicultura, 31(2): 143 152.

Bello S, Assogba-Komlan KF, Baco MN. 2012. Guide pour la production de l'oignon dans l'Alibori. INRABCRAN/AFD/PADSE, Bénin, p. 53.

Bello S. 2012. Analyse diagnostique de la production et de la commercialisation de l'oignon de 1995 à 2009 au Nord-Est du Bénin. INRAB, Bénin, p. 35.

Blackshaw RE, Brandt RN, Brandt H, Janzen HH, Toby E. 2004. La réponse des espèces contre les mauvaises herbes à la fertilisation en phosphore. Weed Science Society of America (WSSA), 52(3): 406 412. DOI: https://doi.org/10.1614/WS03-122R.

Carlson HL, Kirby D. 2005. Effect of Herbicide Rate and Application Timing on Weed Control in Dehydrator Onions. University of Florida, Intermountain Research and Extension Center, 115p.

César C, Franck B. 2006. Évaluation de nouveaux herbicides dans la carotte, le céleri, le chou chinois, la laitue et l'oignon en sols organiques. Ministère de l'Agriculture, des Pêcheries et de l'Alimentation.

Conn KE, Lutton JS, Rosenberger SA. 2012. Onion: Disease Guide. A practical guide for seedmen, growers and agricultural advisors. Seminis Grow forward, p. 69.

Currah L. 2002. Onions in the tropics: cultivars and country reports. In Allium Crop Science: Recent Advances, Rabinwitch HD, Currah L (ed). CABI Publishing: Wallingford UK, New York, USA; 379 - 408.

Davis RM, Aegerter BJ. 2010. Pest Management Guidelines: Onion Garlic. University of California Integrated Pest Management (UCIPM), p. 30.

De Lannoy G. 2001. Oignon Alliums cepa L. In Agriculture en Afrique tropicale, Raemaekers RH (ed). DGCI: Bruxelles; 518 - 526.

Delamarre C. 2010. Fiche technique oignon blanc Biologique. Chambre d'Agriculture de Lot et Garonne, p. 2.

DGESS (Direction des Etudes et des Statistiques Sectorielles). 2015. "Résultats définitifs de la campagne 
agricole et de situation alimentaire et nutritionnelle". DGESS-MAAH, p. 77.

DPSAA (Direction de la prospective et des statistiques agricoles et alimentaires). 2011. Rapport d'analyse du module maraîchage. DPSAA, Ouagadougou, Burkina Faso, p. 214.

EASYPol. 2007. Ressources pour l'élaboration des politiques: Analyse de la filière maraichage au Burkina Faso. EASYPol, Burkina Faso. www.fao.org/easypoldu 08/07/2013.

Garané A, Some K, koussoube S, Traore M, Sawadogo M. 2017b. Flore adventice de la patate douce (Ipomea patatas (L) Lam) du centre du Burkina Faso: structure et fréquence des espèces. Afrique Science, 13(4) :381-399.

http://www.afriquescience.info.

Garané A, Some K, Traore M, Sawadogo M. 20017a. Impacts du mode de désherbage de l'oignon sur les caractéristiques floristiques, la densité et la biomasse adventices du centre du Burkina Faso. Journal of Animal \&Plant Sciences, 34(3): 5491-5508. http://www.m.elewa.org/JAPS

Ghosheh HZ. 2004. Single Herbicide Treatments for Control of Broadleaved Weeds in Onion (Allium cepa L.). Crop Protection, (23): 539-542. DOI : https://doi.org/10.1016/J.cropro.2003.10. 010.

Gourc D, Monnier D, Payet JD. 2007. Oignon. In Guide Pratique pour l'Ile de la Réunion, Union Européenne (éd). Armeflhor, Odeadom: Département de la Réunion ; 106.

Gouyahali S, Raymond K, Siédouba GY. 2016. Analyse des systèmes de production de l'oignon bulbe autour du barrage de Goinré dans la province du Yatenga au Nord du Burkina Faso. Int. J. Biol. Chem. Sci., 10(3): 1173-1183. DOI: http://dx.doi.org/10.4314/ijbcs.v10i3.22.

Hodonou-Gotoechan H, Savi AD. 2001. Etude diagnostique sur la production de l'oignon dans le département de
l'Alibori. Actes de l'Atelier Scientifique Nord Edition 2001, Parakou, Bénin, pp. $109-119$.

Hüsrev M, Doğan I. 2003. Invasive weed species in onion production systems during the last 25 years in Amasya, Turkey. Pak. J. Bot., 35(2): 155-160. www.pakbs.org/pjbot/PDFs/35(2)/PJB35 (2)155.pdf

Kurtz C. 2013. Productivity and conservation of onions affected by nitrogen fertilization in no-tillage system. Hortic. Bras., 31(4): 559 - 567.

Le Bourgeois T, Merlier H.1995. "Adventrop: Les Adventices d'Afrique Soudano-Sahélienne. CIRAD-CA : Monpellier, France.

Mario L. 2015. Utilisation des herbicides dans l'oignon semé en sol organique. Agriculture, Pêcherie et Alimentation.

Marnotte P. 1984. "Echelle de la commission des Essais Biologiques (CEB)". Échelle revue en 1984. Fiches techniques CIRAD, p. 4.

Merlier AH, Montegut J. 1982. Adventices tropicales. Ministère des relations extérieures, Coopération et Développement, Paris.

Messiaen CM, Rouamba A. 2004. Allium cepa L. In PROTA (Plant Resources of Tropical), Grubben GJH, Denton OA (Ed). Wageningen: Netherlands. http:// www.prota4u.org/search.asp.

Moussa A. 2013. Désherbages des cultures maraîchères au Niger. Rapport d'activité 2012, INRAN, p. 11.

Nasreen S, Haque MM, Hossain MA, Farid ATM. 2007. Nutrient uptake and yield of Onion as influenced by nitrogen and sulphur fertilization. Bangladesh $J$. Agric. Res., 32(3): 413-420.

Ngailo S, Shimelis H, Sibiya J, Mtunda K. 2013. Sweetpotato breeding for resistance to sweetpotato virus disease and improved yield: progress and challenges. African journal of agricultural research, 8(25): 3202-3215. DOI: 10.5897/AJAR12.1991 
PAFASP. 2011. Compte rendu de l'atelier nationale du bilan de la campagne 20092010 et Programmation 2010-2011, de la filière oignon dans la zone d'intervention du PAFASP. PAFASP, p. 84. http://www.pafasp.org/oignon.

Qasem JR. 2006. Chemical. Weed Control in Seedbed Sown Onion (Allium cepa L.). Crop Prot., 25: 618-622. DOI: https://doi.org/10.1016/J. cropro.2005.09.008.

Smith R, Fennimore SA. 2008. Management guidelines: Onion and Garlic weeds" and Natural Resources. Flint ML (Ed): University of California Publication; 34 53.

Stahnke G. 2008. Turfgrasses Weed Control: Influence of Cultural Practices on Weed Encroachment. PNW Weed Management Handbook: Turfgrasses.
Thiombiano A. 2005. "Les combretaceae du Burkina Faso: taxonomie, écologie, dynamique et régénération des espèces". Thèse de Doctorat d'État ès Sciences Naturelles, Univ. de Ouagadougou, p. 290.

Uygur S, Gürbüz R, Uygur FN. 2010. Weeds of onion fields and effects of some herbicides on weeds in Cukurova region, Turkey. African Journal of Biotechnology, 9(42):7037-7042. DOI: https://doi.org/10.5897/AJB10.1005.

Zakari AH, Mahamadou CHI, Toudou A. 2015. Effet de l'azote sur l'aptitude à la conservation des bulbes d'oignon (Allium cepa L.). Int. J. Biol. Chem. Sci., 9(6): 2889-2896. DOI: http://dx.doi.org/10.4314/ijbcs.v9i6.28. 